Reprod. Nutr. Develop., 1988, 28 (3 B), 839-844

\title{
Action des protéinases musculaires sur les myosines rapide et lente. Relation avec la protéolyse post-mortem dans des muscles de type contractile variable
}

\author{
A. OUALI, E. DUFOUR, A. OBLED, Christiane DEVAL, C. VALIN
}

Station de Recherches sur la Viande. I.N.R.A., Theix, 63122 Ceyrat, France.

Summary. Effect of muscle proteinases on fast and slow myosins. Relationship with post-mortem proteolysis in muscles of variable contractility.

Sensitivity to proteolysis of fast and slow rabbit skeletal muscle myosins with cathepsins $\mathrm{D}, \mathrm{B}, \mathrm{H}$ and $\mathrm{L}$ and with calpains $\mathrm{I}$ and $\mathrm{II}$ was studied in various incubation conditions. Cathepsins D, B and $L$ degraded both myosins and exhibited different specificities towards these proteins. Moreover, in each case slow myosin appeared to be less sensitive to proteolysis than fast myosin. This finding agrees well with the lower extent of protein hydrolysis observed during post-mortem ageing of meat in slow twitch pork muscles.

\section{Introduction.}

Dans la cellule musculaire, la présence de systèmes protéolytiques efficaces est indispensable au bon fonctionnement du turnover protéique (Mayer et Dehorty, 1986). A ce jour, deux systèmes ont été particulièrement bien décrits. Le système lysosomal actif à $\mathrm{pH}$ acide et constitué d'une carboxyl protéinase, la cathepsine $D$, et de plusieurs cystéine protéinases dont les plus importantes sont les cathepsines $\mathrm{B}, \mathrm{H}$ et $\mathrm{L}$. Le second, actif à $\mathrm{pH}$ neutre, comprend deux cystéine-protéinases calcium dépendantes connues sous le nom de calcium activated neutral proteinase (CaANP) ou de calpaine qui ne diffèrent que par leurs exigences en calcium.

Après la mort des animaux de boucherie, ces systèmes protéolytiques sont également sollicités puisqu'ils semblent impliqués dans le processus de maturation des viandes (Valin, 1985). En effet, la transformation du muscle en viande se traduit principalement par une amélioration progressive de la tendreté de ce tissu, consécutive à la fragilisation de la structure contractile par ces endopeptidases, le collagène n'étant pas affecté. La maturation est un processus qui varie, à la fois en vitesse et en intensité, entre muscles d'un même animal en relation avec leur typage contractile et métabolique. C'est pour tenter de préciser les causes de 
cette variabilité que nous avons entrepris cette étude de l'action des cathepsines $\mathrm{D}, \mathrm{B}, \mathrm{H}$ et $\mathrm{L}$ ainsi que des calpaines sur des myosines de muscles de lapin à contraction rapide ou lente. Les résultats sont discutés par rapport à l'intensité de la protéolyse, en cours de maturation, dans des muscles de porc de type contractile différent.

\section{Matériel et méthodes.}

La myosine a été préparée selon la méthode de Offer (1973) à partir de muscles de lapin qui sont, soit le muscle Psoas major constitué essentiellement de fibres de type lla (myosine rapide), soit le muscle Semimembranosus proprius constitué de fibres de type I (myosine lente) ; les muscles étant prélevés environ 10 min après la saignée.

Les cathepsines $B, H, L$ et $D$, extraites à partir de lysosomes de foie de rat pour les trois premières et de muscle de bceuf pour la dernière, ont été partiellement purifiées selon les méthodes précédemment décrites (Obled et al., 1984 ; Dufour et al., 1987). Les calpaines I et II, obtenues à partir de muscles de lapin, étaient testées après fractionnement par chromatographie sur DEAE-Séphacel selon la méthode de Vidalenc et al. (1983). L'activité hydrolytique des cathepsines $B, H$ et $L$ était mesurée selon la méthode de Barrett (1980). La cathepsine D était testée en présence d'hémoglobine comme substrat (Ouali et Valin, 1981). Pour les cystéine-protéinases, une unité d'enzyme (U) libère $1 \mu \mathrm{mole}$ de $\mathrm{NMec} / \mathrm{ml} / \mathrm{min}$; pour la cathepsine $D$ et les calpaines une unité augmente l'absorption à $280 \mathrm{~nm}$ du surnageant de 0,001 Unité de D.O. par heure et par min respectivement.

La myosine, sous forme insoluble $(6-7 \mathrm{mg} / \mathrm{ml})$, était incubée à $25^{\circ} \mathrm{C}$ et à $\mathrm{pH} 6,0$, en tampon Tris-acétate $0,05 \mathrm{M}$ en présence de DTT $2 \mathrm{mM}$ dans le cas des cystéine protéinases pendant des temps variables. Dans le milieu d'incubation était ajouté soit du $\mathrm{MgCl}_{2}$ ou du $\mathrm{CaCl}_{2}$ lorsqu'il s'agissait des calpaines, soit de I'EDTA à une concentration finale de $2 \mathrm{mM}$. Les quantités d'enzyme $(\mathrm{U} / \mathrm{mg}$ de myosine) étaient de 0,32 U pour la cathepsine $D$ et de 0,01 U, 0,001 U et 0,007 U, respectivement, pour les cathepsines $\mathrm{B}, \mathrm{H}$ et $\mathrm{L}$. En ce qui concerne les calpaines, la concentration variait entre 20 et $150 \mathrm{U}$. L'arrêt de la réaction était réalisé par addition d'iodoacétate à une concentration finale de $1 \mathrm{mM}$ (cathepsines $\mathrm{B}, \mathrm{H}$ et L) ou de pepstatine $0,1 \mathrm{mM}$ (cathepsine D) ou d'EDTA $5 \mathrm{mM}$ (calpaïnes). L'hydrolyse de la myosine par la chymotrypsine et la purification des fragments libérés ont été réalisées selon la méthode de Morgassian et Lowey (1983).

Pour le suivi de la protéolyse intramusculaire dans la viande en cours de maturation, les muscles Longissimus dorsi (LDp) (type Ila), Semitendinosus (STp) (intermédiaire) et Trapezius (TZp) (type I) de porc, prélevés $1 \mathrm{~h}$ après la saignée et conditionnés sous vide, étaient maintenus à $10^{\circ} \mathrm{C}$ jusqu'au lendemain matin puis conservés ensuite à $2{ }^{\circ} \mathrm{C}$ pendant 9 jours. La préparation des myofibrilles selon la méthode de Bendall (1961) était réalisée sur des prélèvements à $2 \mathrm{~h}$ post-mortem puis après 1,3 et 9 jours de stockage. Après chaque 
prélèvement, les muscles étaient remis sous vide et replacés à $2{ }^{\circ} \mathrm{C}$. L'électrophorèse de la myosine et des myofibrilles a été réalisée selon la méthode de Laemmli (1970).

\section{Résultats et discussion.}

\section{Action des différentes protéinases sur la myosine rapide.}

De nombreux travaux ont montré que I'hydrolyse ménagée de la myosine par différentes protéinases libère des fragments presque identiques et relativement bien définis: heavy méromyosine (HMM), light méromyosine ( $L M M)$, rod ou batonnet, HMM-subfragment 1 (S1) et HMM-subfragment 2 (S2). La chymotrypsine dégrade ainsi la chaîne lourde $(\mathrm{HC})$ soit en HMM et LMM soit en rod et S1 selon que l'incubation ait été réalisée en présence d'EDTA ou de $\mathrm{MgCl}_{2}$ respectivement (fig. $1 \mathrm{~A}-\mathrm{CHY}$ ). En présence de $\mathrm{MgCl}_{2}$, la chaîne légère $\mathrm{LC} 2 \mathrm{f}$ n'est que partiellement hydrolysée tandis qu'elle l'est totalement dans l'autre cas. La cathepsine $D$ présente la même spécificité d'action que la chymotrypsine (fig. 1A-CD) tout au moins vis-à-vis de la HC puisque la LC2f est pratiquement intacte, les autres chaînes légères LC1f et LC3f étant résistantes à l'action de ces deux protéinases.

Que l'incubation soit conduite en présence d'EDTA ou de $\mathrm{MgCl}_{2}$, la cathepsine $B$ hydrolyse la $H C$ à la jonction HMM-LMM tandis que les trois chaînes légères $L C 1 f, L C 2 f$ et $L C 3 f$ ne sont pratiquement pas affectées (fig. $1 \mathrm{~A}$ -CB). L'action limitée de cette protéinase sur la HC confirme les résultats de Schwartz et Bird (1977). En présence d'EDTA, la cathepsine L dégrade la HC en trois fragments majeurs : rod, S1 et LMM (fig. 1A-CL). Nous notons également l'apparition de S2 long et de S2 court, ce dernier étant plus abondant. Les mêmes produits d'hydrolyse sont observés lorsque l'EDTA est remplacé par du $\mathrm{MgCl}_{2}$ excepté que le fragment S2 long est totalement absent. Dans tous les cas nous notons la dégradation complète des chaînes légères. Bien que les cathepsines $B$ et $L$ soient toutes deux des cystéine protéinases, elles présentent des spécificités et des capacités hydrolytiques différentes vis-à-vis de la myosine. La cathepsine $\mathrm{H}$ et les calpaines qui sont également des cystéines protéinases sont, par contre, sans effet sur cette protéine.

\section{Action des différentes protéinases sur la myosine lente.}

Comme le montre la figure $1 \mathrm{~B}$, toutes ces protéinases coupent la myosine lente à des sites très voisins de ceux observés pour la myosine rapide. Toutefois, il apparaît nettement que, dans le cas de la myosine lente, la préservation, par $\mathrm{MgCl}_{2}$, de certains sites très sensibles à la protéolyse, est beaucoup moins marquée puisque les produits de dégradations sont pratiquement les mêmes que I'on soit en présence d'EDTA ou de $\mathrm{MgCl}_{2}$. Ceci peut s'expliquer soit par le fait 


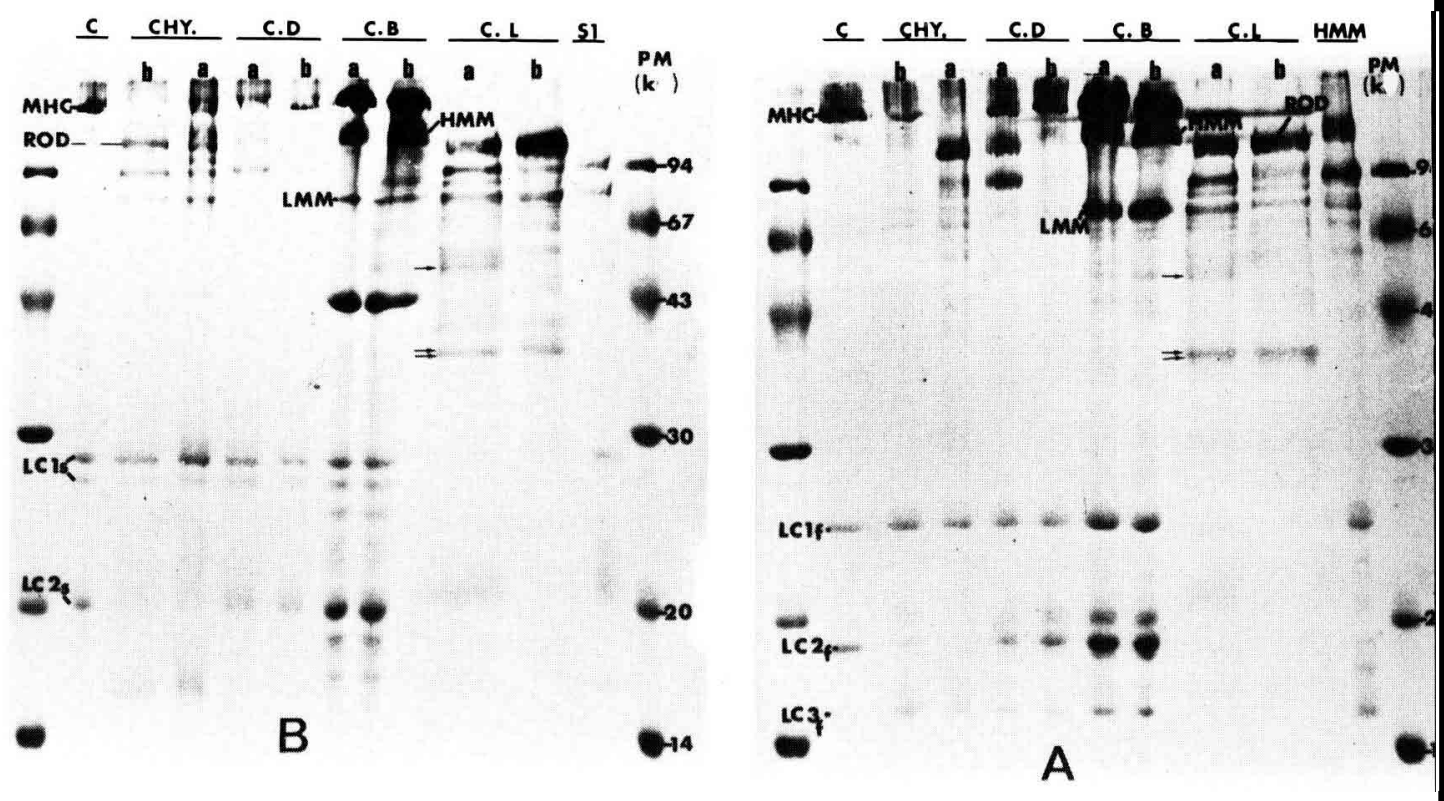

FIG. 1 - Profil électrophorétique de l'hydrolysat de myosine rapide (A) et lente (B), après action de la chymotrypsine (CHY) et des cathepsines $\mathrm{D}(\mathrm{CD}), \mathrm{B}(\mathrm{CB})$ et $\mathrm{L}(\mathrm{CL})$ en présence d'EDTA (a) ou $\mathrm{MgCl}_{2}$ (b) ainsi que des myosines non incubées (C). Les marqueurs de poids moléculaire (PM) sont: phosphorylase b $(94 \mathrm{k})$, serum albumine bovine $(67 \mathrm{k})$, ovalbumine $(43 \mathrm{k})$, anhydrase carbonique $(30 \mathrm{k})$, soybean trypsin inhibitor $(20,1 \mathrm{k})$ et $\alpha$-lactalbumine $(14,4 \mathrm{k})$. Les abbréviations utilisées sont: MHC (myosin heavy chain), HMM (high meromyosin), LMM (light meromyosin), LCf (fast myosin light chains), LCs (slow myosin light chains). L'échantilion HMM correspond aux fragments HMM et $\mathrm{S} 1$ purifiés obtenus par action de la chymotrypsine. Les fragments $\mathrm{S} 2$ sont indiqués par une simple ( $\mathrm{S} 2 \mathrm{long}$ ) ou une double ( $\mathrm{S} 2$ court) flèche.

que ce dernier induise ici un changement de conformation moins accentué que dans la myosine rapide laissant ainsi le site de coupure à la jonction S1 -S2 encore très accessible aux protéinases, soit par une position différente de ce site qui serait, au niveau de la myosine lente, plus proche de l'extrémité $C$ terminale que dans le cas de la myosine rapide. Au vu de nos résultats et de ceux de Bugaiski et al. (1983) montrant que le fragment $\mathrm{S} 1$ obtenu à partir de myosine lente présente un poids moléculaire supérieur à celui généré à partir de myosine rapide, la seconde hypothèse paraîtrait la plus probable mais ceci reste à vérifier.

Une autre différence notable qui conforte les observations de Koretz (1983) concerne la vitesse d'hydrolyse de la myosine lente par la cathepsine $L$ qui est quatre fois plus faible que celle de la myosine rapide (résultats non publiés).

\section{Variabilité de l'intensité de la protéolyse dans divers muscles de porc.}

Post-mortem, l'action des protéinases se traduit par l'apparition de plusieurs produits de dégradation, en particulier dans la zone de poids moléculaire comprise entre 25000 (LC1f) et 34000 (tropomyosine) (Ouali et al., 1983). Au niveau du muscle LDp, nous voyons apparaître une bande de PM 30000 migrant 


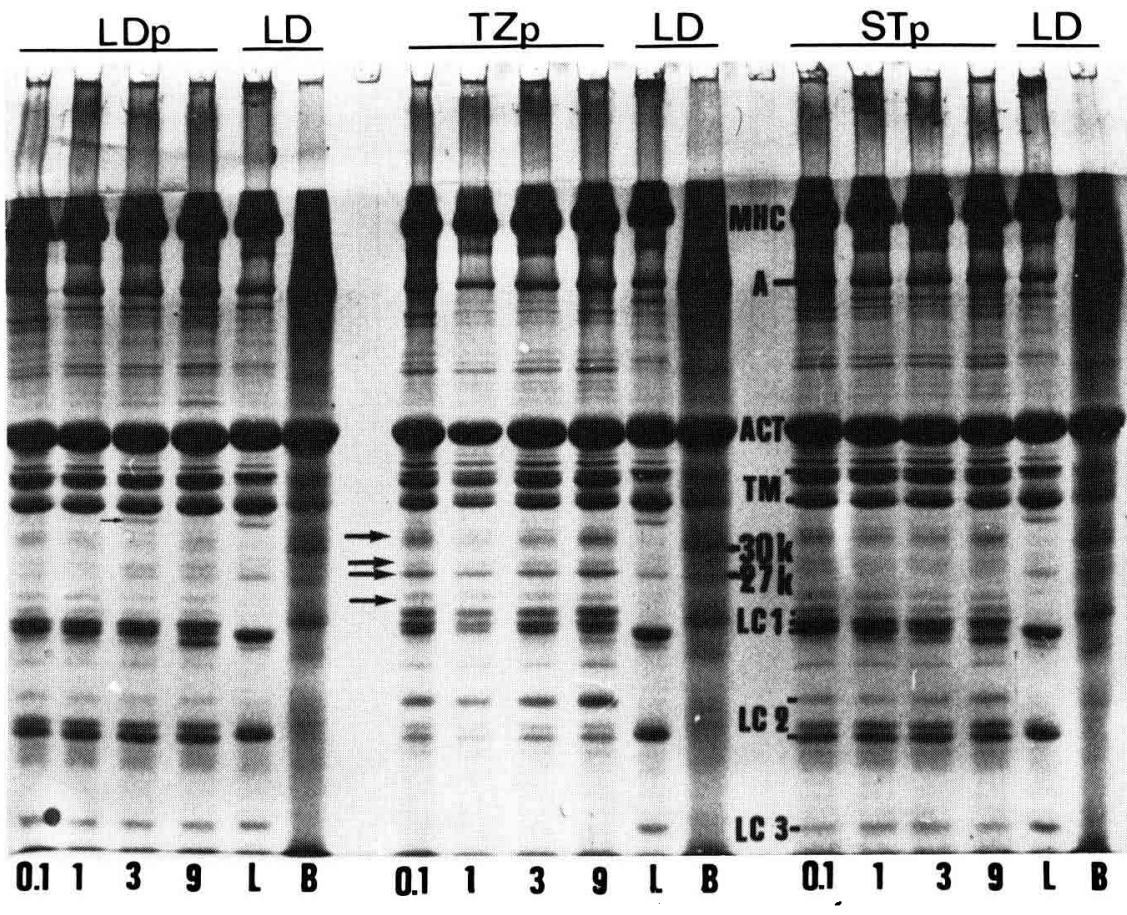

FIG. 2. - Profil électrophorétique de myofibrilles des musc/es Longissimus dorsi (LDp), Trapezius (TZp) et semitendinosus (STp) de porcs conservés à $+2{ }^{\circ} \mathrm{C}$ pendant $0,1,3$ et 9 jours. A titre de comparaison, des myofibrilles du muscle Longissimus dorsi (LD) de lapin (L) et de bovin (B) conservé pendant 9 jours à $+2{ }^{\circ} \mathrm{C}$ ont été chargées. Les abbréviations utilisées sont: MHC (myosin heavy chain), A ( $\alpha$-actinin), ACT (actine), TM (tropomyosine) et LC (myosin light chain). Les flèches indiquent les différentes bandes de PM compris entre 25 et $34 \mathrm{k}$ dont il est question dans le texte.

comme le composé majoritaire observé dans le muscle LD de bovin (LD-B) (fig. 2). Nous notons, par ailleurs, dès la mort de l'animal, la présence de plusieurs autres composés, de nature indéterminée, dont la concentration reste pratiquement constante au cours du temps et qui ne semblent pas exister dans les muscles de lapin (LD-L) et de bovin. Le premier, qui migre juste avant la chaîne légère $\mathrm{LC} 1 \mathrm{a}$, est décelable dans les trois muscles considérés à un taux très voisin. Le second migre comme le produit de dégradation majeur apparaissant dans le LD de lapin de PM 27 000. Par comparaison aux muscles LDp (type lla) et STp (type intermédiaire), sa concentration paraît nettement supérieure dans le muscle TZp (type l). Ce dernier composé pourrait correspondre à l'anhydrase carbonique qui est caractéristique des fibres de type I (Staron et Pette, 1987) mais qui existe également dans les fibres de type II. Le troisième, de PM voisin de 32000 , est présent dans les trois muscles mais semblerait aussi plus abondant dans le TZp que dans les deux autres. Nous notons enfin, juste au-dessous de la tropomyosine, l'apparition, après 3 jours de conservation, d'une bande qui disparaît ensuite entre le troisième et le neuvième jour. Cette bande, qui n'est présente à aucun moment dans les muscles TZp et STp, est encore très marquée dans le LD de lapin 9 jours post-mortem. Dans le cas des muscles TZp et STp, nous n'observons que 
très peu de modifications dans cette zone ce qui semblerait indiquer que la protéolyse est plus intense dans le muscle LDp qui est de type lla. Ces résultats semblent confirmer la variabilité de l'intensité de protéolyse observée dans le cas des myosines en fonction de leur typage et suggèreraient un rôle essentiel du type contractile des muscles dans la définition des caractéristiques cinétiques du processus de maturation.

$13^{e}$ Réunion du groupe Développement I.N.R.A. Cap d'Agde, 25-27 mai 1987.

\section{Références}

BARRETT A. J., 1980. Fluorimetric assays for cathepsins $\mathrm{B}$ and $\mathrm{H}$ with methylcoumarylamide substrates. Biochem. J., 187, 909-912.

BENDALL J. R., 1961. A study of the kinetics of the myofibrillar ATPase of rabbit skeletal muscle. Biochem. J., 81, 520-535.

BUGAISKI L. B., BUTLER-BROWNE G. S., SELL S. M., WHALEN R. G., 1984. Structural differences in the subfragment 1 and rod portions of myosin isozymes from adult and developing rat skeletal muscles. J. biol. Chem., 11, 7212-7218.

DUfOUR E., OBLED A., VALIN C., BECHET D., RIBADEAU-DUMAS B., HUET J. C., 1987. Purification and amino acid sequence of chicken liver cathepsin L. Biochemistry, 26, 5689-5695.

KORETZ J. F., 1983. Hybridization and reconstitution of thick filament structure. Methods Enzymol., 85B, 20-55.

LAEMMLI U.K., 1970. Cleavage of structural proteins during the assembly of the head of bacteriophage T4. Nature, 227, 680-685.

MAYER R. J., DOHERTY F., 1986. Intracellular protein catabolism : state of the art. FEBS Lett., 198, 181 - 193.

MORGASSIAN S. S., LOWEY S., 1983. Preparation of myosin and its subfragments from rabbit skeletal muscle. Methods Enzymol., 85B, 55-71.

OBLED A., OUALI A., VALIN C., 1984. Cysteine proteinase content of rat muscle lysosomes. Evidence for an unusual proteinase activity. Biochimie, 66, 609-616.

OFFER G., 1973. A new protein of the thick filaments of vertebrate skeletal myofibrils. J. mol. Biol., 74, 653-676.

OUALI A., OBLED A., COTTIN P., MERDACI N., DUCASTAING A., VALIN C., 1983. Comparative effects of post mortem ageing and $\mu \mathrm{M}$ CaANP on bovine and rabbit myofibrillar proteins. $J$. Sci. Food Agric., 34, 466-476.

OUALI A., VALIN C., 1981. Effect of lysosomal enzymes and CaANP on the myofibrillar ATPase activities. Relationship with the ageing changes. Meat Sci., 5, 233-245.

SCHWARTZ W. N., BIRD J.W. C., 1977. Degradation of myofibrillar proteins by cathepsins B and D. Biochem. J., 167, 811-820.

STARON R. S., PETTE D., 1987. The multiplicity of combinations of myosin light chains and heavy chains in histochemically typed single fibres. Biochem. J., 243, 695-699.

VALIN C., 1985. Les protéases des viandes, 279-312, In MOURANCHE A., COSTES C., Hydrolases et dépolymérases, Gauthier-Villars, Paris.

VIDALENC P. L., COTTIN P., MERDACI N., DUCASTAING A., 1983. Stability of two $\mathrm{Ca}^{++}$ dependent proteinases and their specific inhibitor during post-mortem storage of rabbit skeletal muscle. J. Sci. Food Agric., 34, 1241-150. 